EPJ Web of Conferences 112, 03002 (2016)

DOI: $10.1051 /$ epjconf/201611203002

(C) Owned by the authors, published by EDP Sciences, 2016

\title{
Nuclear Parton Distributions with the LHeC
}

\author{
Max Klein ${ }^{1,2, a}$ \\ ${ }^{1}$ University of Liverpool, Department of Physics, L697ZE Liverpool, Oxford Street, United Kingdom \\ ${ }^{2}$ CERN, 1211 Geneva 23, Switzerland
}

\begin{abstract}
Nuclear parton distributions are far from being known today because of an infant experimental base. Based on design studies of the $\mathrm{LHeC}$ and using new simulations, of the inclusive neutral and charged current cross section measurements and of the strange, charm and beauty densities in nuclei, it is demonstrated how that energy frontier electron-ion collider would unfold the complete set of nuclear PDFs in a hugely extended kinematic range of deep inelastic scattering, extending in Bjorken $x$ down to values near to $10^{-6}$ in the perturbative domain. Together with a very precise and complete set of proton PDFs, the LHeC nPDFs will thoroughly change the theoretical understanding of parton dynamics and structure inside hadrons.
\end{abstract}

\section{Introduction}

Nuclear parton distribution functions (nPDF) are considered through the factorisation theorem [1] of pQCD as universal descriptions of ion-ion ( $p A$ or $A A$ ) and lepton-hadron ( $l A)$ interactions [2]. They are currently poorly determined while the physics of $A A$ scattering with RHIC and the LHC has made striking progress. The theory of nuclear structure, of parton dynamics inside nuclei and of the evolution of the Quark-Gluon Plasma state require a much deeper understanding of nPDFs than has been possible to achieve, also including available LHC $p P b$ data [3], which to much surprise even did exhibit collective phenomena.

PDFs are determined in deep inelastic lepton-hadron scattering, with sometimes useful constraints derived from hadron-hadron interactions. The situation of nPDFs is that unsatisfactory because HERA was not operated with ion beams despite serious proposals [4] to make that happen. Therefore, one is in a situation which resembles the status ante HERA in the determination of proton PDFs: the gluon is essentially unknown, heavy quark distributions are undetermined, up and down light quarks only marginally distinguished and the range in Bjorken $x$ and 4-momentum transfer squared, $Q^{2}$, is very limited. These experimental deficiencies are particularly disturbing because the nucleus, unlike the proton, is a much more complex environment for the dynamics of partons to unfold. The nuclear PDFs, $f_{A}^{i}\left(x, Q^{2}\right)$, comprise not only the genuine probabilities for the parton densities but account as well for nuclear binding effects known, for decreasing $x$, as Fermi motion, EMC effect, anti-shadowing and shadowing. It therefore is not only desirable but necessary to determine the full set of PDFs, $i=x g, u_{v}, d_{v}, \bar{u}, \bar{d}, s, c$ and $b$, with nuclei as well as with protons in order to then determine experimentally, with $\mathrm{pQCD}$ assisting, the ratio $R_{A}^{i}=f_{p}^{i} / f_{A}^{i}$ parton by parton in a maximum

a e-mail: max.klein@cern.ch 
range of $x$ and $Q^{2}$. That is the core of the $e A$ measurement programme of the LHeC. Special attention is also given to electron-deuteron scattering because the deuteron is a very special nucleus providing the probably cleanest information on the structure of the neutron.

This brief summary, presented at the POETIC workshop at Paris in September 2015, relies on the layout of the $e A$ programme in the LHeC Conceptual Design Report (CDR) [5], see also the $\mathrm{LHeC}$ web page [6] for subsequent presentations. The present contribution also illustrates a new attempt to fit nPDFs based on newly simulated $\mathrm{LHeC}$ cross section data as well as novel simulations of the determination of the strange and heavy quark PDFs in nuclei. The $\mathrm{LHeC}$ is designed to collide electrons of $60 \mathrm{GeV}$ beam energy off protons of $7 \mathrm{TeV}$ energy (at luminosities up to $1 \mathrm{ab}^{-1}$ ) and off lead $(\mathrm{A}=207, \mathrm{Z}=82)$ ions of $2.7=7 \cdot \mathrm{Z} / \mathrm{A} \mathrm{TeV}$ energy (at an estimated integrated luminosity corresponding to $\left.1 \mathrm{fb}^{-1}\right)$. This opens the prospect for an unprecedented new base and range of unfolding nuclear PDFs and determining the various nuclear binding effects individually for each parton. At this collider one will also be able to control and exclude higher twist effects which, appearing $\propto 1 / Q^{2}$, result from final state rescattering effects and are likely to be amplified $\propto A^{1 / 3}$ disturbing the determination of nPDFs from low energy experiments even more.

This contribution is organised as follows: Section 2 summarises the status of the current nPDF determinations and presents a summary of the $\mathrm{LHeC}$ data simulation. Section 3 briefly summarises initial results of a study of the determination of PDFs in electron-deuteron scattering. Section 4 presents the nPDF simulation using $\mathrm{LHeC}$ data performed within an adapted EPS09 pQCD framework. Section 5 discusses the gluon distribution and the possible search for saturation of the rise of the gluon density towards low $x$. Section 6 includes the determination of the strange, charm and beauty distributions in nuclei from a future $e A$ operation of the LHeC. A brief summary is presented in Section 7 .

\section{2 nPDFs Today and their Derivation from the LHeC}

Figure 1 presents a summary of presently available sets of nuclear parton distributions, from the HKN (2007), EPS (2009), DSSZ (2012) and nCTEQ (2015) groups with their references and basic characteristics. The data used in all sets come from various fixed target DIS experiments and include dilepton data from Drell-Yan hadron-hadron scattering. Most of the sets use also pion production $D A u$ data for constraining the gluon distribution to some extent. There are three main characteristics of these sets: i) the nPDFs are obtained by using a baseline set of proton PDFs to which a parameterised nuclear correction is fitted. That assumes factorisation of the nuclear and genuine PDF properties, and it introduces an undesirable dependence of the nPDFs on the proton PDF base; ii) owing to the restricted data input, the sets provide only a minimum number of nuclear PDFs employing symmetry assumptions, usually just one valence quark, a combined sea quark and the gluon distribution. The recent nCTEQ analysis removed the $u_{v}=d_{v}$ assumption inherent in all previous analyses and, not surprisingly, large variations appeared with a softer up and a harder down quark distribution; iii) as in the so-called global analyses of proton PDFs, the data fitted are not really consistent among each other. Consequently, the analyses introduce some $\chi^{2}$-tolerance criteria and mimic what a one standard deviation may amount to. All three major deficiencies will be overcome through the LHeC.

The $\mathrm{LHeC}$ provides measurements of $e A$ scattering cross sections in the deep inelastic scattering region $Q^{2}>1 \mathrm{GeV}^{2}$ reaching to $Q^{2}$ up to about $5 \cdot 10^{5} \mathrm{GeV}^{2}$ and corresponding $x$ values between a few times $10^{-6}$ and near to $x=1$. This enables the determination of a complete set of nPDFs in electron-lead scattering at the LHC from the inclusive neutral and charged current cross sections with a clean separation of up and down valence and sea quark distributions. The very high $Q^{2}$ which reaches much beyond the $W$ mass squared makes the CC measurements extremely valuable for the separation of different flavours when taken together with the NC, from photon and $Z$ boson exchange. 
Table 1. Summary of assumed uncertainties for future inclusive cross section measurements at the LHeC.

\begin{tabular}{|l|c|}
\hline Source of uncertainty & Error on the source or cross section \\
\hline scattered electron energy scale & $0.1 \%$ \\
scattered electron polar angle & $0.1 \mathrm{mrad}$ \\
hadronic energy scale & $0.5 \%$ \\
calorimeter noise $(y<0.01)$ & $1-3 \%$ \\
radiative corrections & $1-2 \%$ \\
photoproduction background & $1 \%$ \\
global efficiency error & $0.7 \%$ \\
\hline
\end{tabular}

As will be illustrated below, charm tagging in $\mathrm{CC}$ determines the anti-strange quark distribution in a wide kinematic range to typically $10-20 \%$ precision, while charm and beauty tagging in NC provide high precision determinations of $x c$ and $x b$ from nuclei. Using coherent data from just this, one experiment the uncertainties of these nPDFs will follow from a straightforward $\Delta \chi^{2}=1$ criterion. Since, with much larger luminosity, a comparative PDF set will be measured in $e p$ scattering at the LHeC, the ratios $R_{A}^{i}=f_{p}^{i} / f_{A}^{i}$ can be expected to be measured to few or better than percent accuracy as functions of $x$ and $Q^{2}$, separately for all $i$. It is furthermore expected that the nPDFs are not universal in the sense that they depend on the type of boson $\left(\gamma, W^{+}, W^{-}, Z\right)$ with which they are probed [7]. Effects like that may only be experimentally established at DIS energies much exceeding HERA as it requires precision measurements of PDFs with disentangled electromagnetic and weak contributions. It is obvious that the $\mathrm{LHeC} e A$ programme is expected to change the theoretical description of nPDFs in QCD thoroughly, in diversity, accuracy and range.

The QCD analyses illustrated subsequently of pseudo LHeC cross section data employ sets of simulated NC and CC measurements under assumptions which are summarised in Table 1 . The cross section simulation was done numerically employing derivative formulae from [8] and found to compare well to a detailed Monte Carlo simulation when tested for the conditions of the H1 experiment. The assumptions made are all reasonable when comparing with the H1 achievements, which shall probably be exceeded owing to new detector techniques and higher statistics. The control of radiative corrections in $e A$ scattering is a special challenge as these grow $\propto Z^{2}$. The LHeC detector thus needs to be equipped with reliable photon detectors and the exploitation of the energy-momentum conservation, via the $E-p_{z}$ cut, should further reduce the effect of photon radiation to a few per cent level. It is also to be noted that the semi-inclusive measurements of the $s, c$ and $b$ quark distributions carry additional uncertainties for tagging, acceptance and background influences.

\section{Deuteron PDFs from the LHeC}

Electron-deuteron scattering at the $\mathrm{LHeC}$ is of very special interest because it enables to access the neutron structure and study the binding effects in the simplest bound proton-neutron configuration. For the derivation of PDFs, $e D$ is the obvious closest partner to $e p$ scattering in measuring an independent up-down quark combination. This permits to disentangle $u$ and $d$ in the valence and the sea region, assisting and cross checking the effect of adding $\mathrm{CC}$ and $\mathrm{NC}$ data in ep. Figure 2 illustrates the very high precision one would obtain in $e D$ scattering alone, assuming a luminosity of $0.3 \mathrm{fb}^{-1}$ and systematic errors as listed above. It is visible that the gluon and sea distributions are determined to a few per cent precision and with larger relative uncertainty in the valence quark region $x>0.3$ where both are very small. The valence quarks are measured very well in the medium to large $x$ range. At smaller $x<0.01$ their relative uncertainty increases while the distributions fall. Details of a similar fit 
to simulated ep data have been released through [9] and the resulting proton PDFs with their expected full experimental uncertainty are available in the LHAPDF data base.

This remarkable sensitivity is obtained in an $e D$ only study. The combination of $e p$ and $e D$ data resolves any $u-d$ ambiguity, within the uncertainty of nuclear corrections. These, however, can be controlled experimentally by two novel means: i) the high resolution tagging of protons in en scattering and ii) through Gribov's relation of shadowing and diffraction, see [4] for a discussion. It shall also be stressed that in $e D$ a singlet combination of PDFs is measured which stabilises the QCD evolution of PDFs and thus supports the ambitious extraction of $\alpha_{s}$ to per mille accuracy [5]. There is no deuteron beam yet foreseen for the LHC, which, however, is a straightforward project of reasonable effort.

\section{Study of Nuclear PDFs from LHeC eA Pseudodata}

A first analysis has been pursued within the EPS09 framework [10]. It uses the EPS09 data (see Figure 1) and adds for comparison the $\mathrm{LHeC}$ neutral current data, keeping the three basic distributions for both valence, the light sea quarks and the gluon. The analysis uses CTEQ6.6 as baseline and the SACOT heavy quark treatment. The default result is shown as dashed band in Figure 3 (top). Accounting for the fact that there are no $l A$ DIS data available for $x<0.01$ (indicated by the vertical line in $R_{G}^{A}$ ), while the $\mathrm{LHeC}$ would cover $x>10^{-5}$ the assumption on shadowing to approach a constant for $x \rightarrow 0$ was replaced by a functional form, see [11], with three free parameters. This provides a more

\begin{tabular}{|c|c|c|c|c|c|}
\hline item & HKN07 & EPS09 & DSSZ & nCTEQ & LHeC \\
\hline Reference & $\begin{array}{l}\text { Phys. Rev. } \\
\text { C76 (2007) } \\
065207\end{array}$ & $\begin{array}{l}\text { JHEP } 0904 \\
\text { (2009) } 065\end{array}$ & $\begin{array}{l}\text { Phys.Rev. } \\
\text { D85 (2012) } \\
074028\end{array}$ & $\begin{array}{l}\text { arXiv: } \\
1509.00792\end{array}$ & $\begin{array}{l}\text { Workshops } \\
+ \text { this talk } \\
\text { PRD(2030+) }\end{array}$ \\
\hline Order pQCD & LO \& NLO & LO \& NLO & NLO & NLO & NNLO \\
\hline $\mathrm{NC} e+\mathrm{A} / \mathrm{e}+\mathrm{d}$ DIS & $\sqrt{ }$ & $\sqrt{ }$ & $\sqrt{ }$ & $\sqrt{ }$ & $\mathrm{NC}$ \\
\hline Drell-Yan II in $p+A / p+d$ & $\sqrt{ }$ & $\sqrt{ }$ & $\sqrt{ }$ & $\sqrt{ }$ & -- \\
\hline RHIC pions in $d+A u / p+p$ & & $\sqrt{ }$ & $\sqrt{ }$ & $\sqrt{ }$ & -- \\
\hline Neutrino-nucleus DIS & & & $\sqrt{ }$ & & $\mathrm{CC}$ \\
\hline$\sqrt{ } \mathbf{Q}^{2}$ cut in DIS & $1 \mathrm{GeV}$ & $1.3 \mathrm{GeV}$ & $1 \mathrm{GeV}$ & $2 \mathrm{GeV}$ & free \\
\hline \# of data points & 1241 & 929 & 1579 & 740 & many \\
\hline Free parameters & 12 & 15 & 25 & 17 & $O(20)$ \\
\hline Error sets available & & $\sqrt{ }$ & $\sqrt{ }$ & $\sqrt{ }$ & (y) \\
\hline Error tolerance $\Delta \mathrm{x}^{2}$ & 13.7 & 50 & 30 & 35 & 1 \\
\hline Baseline & MRST98 & CTEQ6.1 & MSTW08 & CTEQ6M & $\begin{array}{l}\text { None - or } \\
\text { ep+eD+eA }\end{array}$ \\
\hline Heavy quark treatment & ZM_VFNS & ZM_VFNS & GM_VFNS & GM_VFNS & s,c,b data \\
\hline
\end{tabular}

Figure 1. Sets of available nuclear parton distributions and, right column, characteristics of the future LHeC determination of nPDFs. 

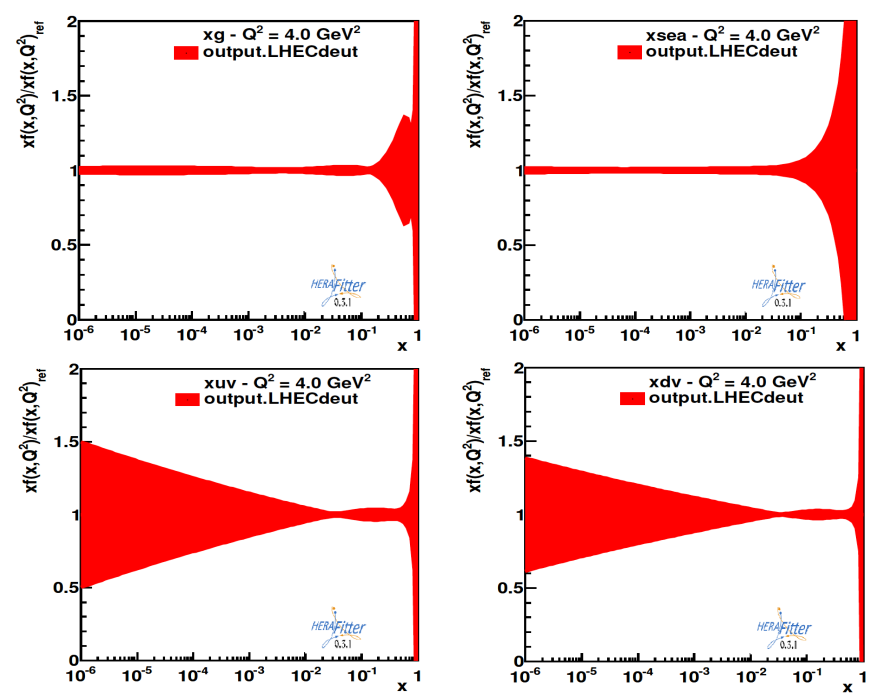

Figure 2. Gluon (left top), sea (right top) and valence quark (down) distribution uncertainties, comprising statistical and experimental uncertainties, from a simulation of electron-deuteron scattering, see text.

appropriate illustration on the inferior control of all $R^{A}$ 's at small $x$, given by the outer error bands presented in Figure 3. The addition of the LHeC NC data, obtained in $e^{-} A$ scattering as characterised in Section 2, leads to a striking improvement on all ratios, especially for the sea and gluon distributions. This illustration is only a first step in a detailed study of the potential for determining nPDFs with the LHeC. It had not included the CC data and neither the direct determinations of the heavy quark distributions. Moreover, as illustrated for the case of deuterons above, with such a complete data set, as will be provided with the $\mathrm{LHeC}$, the $\mathrm{nPDF}$ determination will not need to employ a proton PDF base anymore but rather and directly determine the nuclear PDFs $f_{i}^{A}$. Then a ratio $R$ will follow from the independent fits of the complete sets $f_{i}^{A}$ and of $f_{i}^{p}$. Unlike now these flavour dependent ratios will be fixed with data down to below $x=10^{-5}$. In this region novel effects are predicted to exist, such as non-linear parton interactions including a possible saturation [12] of the density of gluons at small $x$.

\section{On the Saturation of the Gluon Density at Low $x$}

The prediction of non-linear parton-parton dynamics has long been made in the BFKL formalism. At small $x$, terms in the expansion containing logarithms of $x$ are calculated to be large and deviations from the linear evolution in $Q^{2}$, as described by the DGLAP equations, were expected to be observed but surprisingly not established at HERA. There was, however, a rise of the sea quark and gluon distributions discovered towards low $x$. HERA had a cms energy squared $s=Q_{\max }^{2}$ available of $10^{5} \mathrm{GeV}^{2}$.

For the determination of the gluon density $x g$ at small $x$ in DIS one needs to have a lever arm available between, about, 2 and $10 \mathrm{GeV}^{2}$ because it is the logarithmic derivative of the structure function $F_{2}\left(x, Q^{2}\right)$ which determines $x g$. HERA thus could probe $x g$ to $x>10^{-4}$, actually twice larger only for one knows from a Taylor expansion of the splitting function that a measurement of $F_{2}$ at 

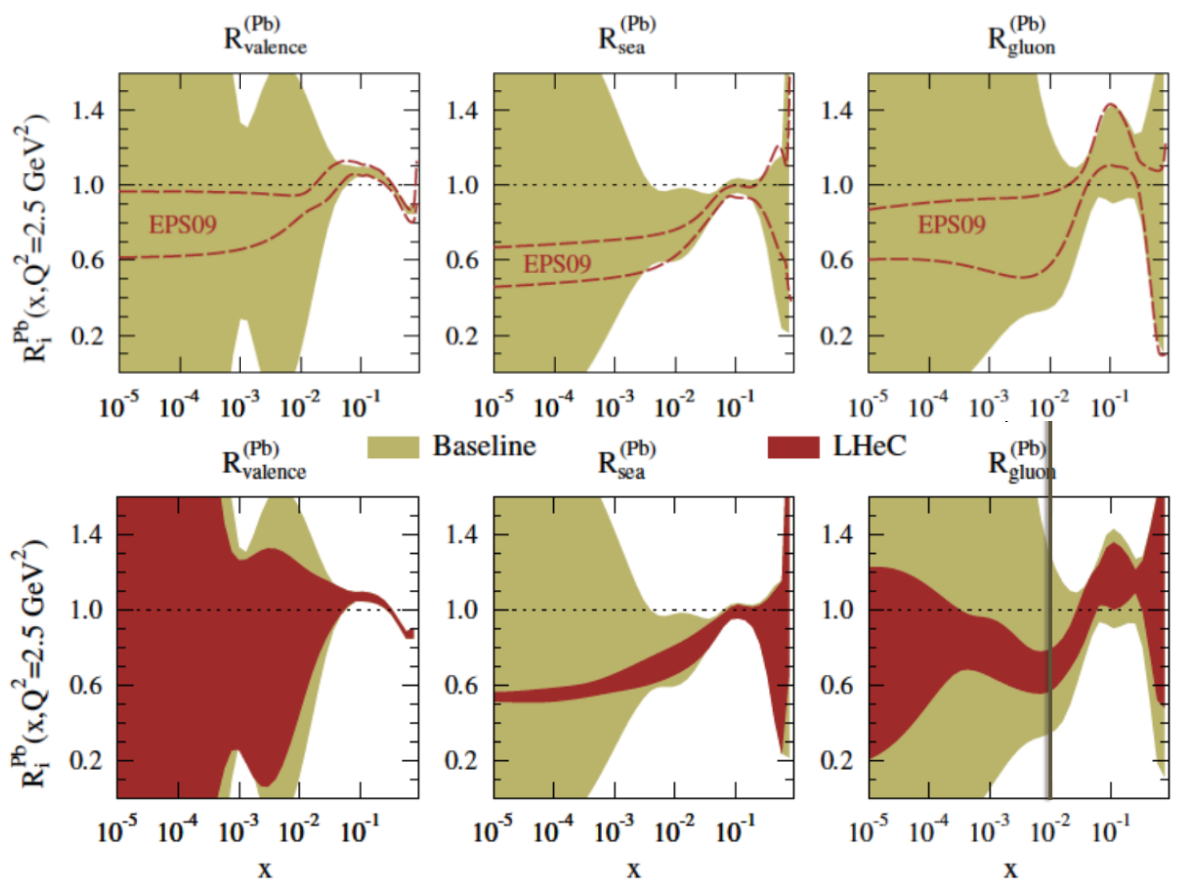

Figure 3. Ratios $R_{i}^{P b}$ of $i=$ valence, sea and gluon distributions (from left to right). Top: inner dashed band: estimated uncertainty of $R$ in the default EPS09 framework; outer area: uncertainty estimate with relaxed assumptions (see text). Bottom: outer area as above. inner dark (red) area: uncertainty band with added pseudo LHeC data (NC only), from [11]. The vertical line in $R_{G}^{P b}$ at $x_{c}=0.01$ indicates that there are no nuclear DIS data available at smaller $x<x_{c}$ which makes the low $x$ uncertainties drawn in the top panel no more than educated guesses with more (dashed) or less (outer bands) strict assumptions on how much nuclear and proton PDFs may be allowed to differ.

certain value of $x$ provides a constraint on $x g$ at $2 x$ [13]. It therefore is required to go to higher beam energies than at HERA if one wanted to establish the effect of gluon saturation ${ }^{1}$ in DIS. The most promising procedure has been studied in the CDR of the LHeC: One needs high precision ep data on $F_{2}$ but also on $F_{L}$ in order to fix the gluon distribution and preventing a simple parameterisation effect to screen a saturation phenomenon. Then one needs to measure $x g$ also in $e A$ scattering, as has been illustrated above. From the three independent $x g$ determinations, using $F_{2}^{p}, F_{L}^{p}$ and $F_{2}^{A}$, one can indeed hope to be able to extract the genuine gluon density $x g\left(x, Q^{2}\right)$, any non-linear corrections and shadowing effects accurately. It should be noted that these measurements and analyses are experimentally and theoretically most demanding and cannot be replaced by interesting control measurements as of diffraction or vector meson production even though these are principally sensitive to $(x g)^{2}$ while resting on theoretically inferior ground.

\footnotetext{
${ }^{1}$ At the low energy EIC one often argues that gluon saturation may be seen in $e A$ scattering for the gluon density was expected to be amplified $\propto A^{1 / 3}$. There are two observations which weaken that argument: i) the gluon density at initial scales $Q^{2}$ and low $x$ is observed to be small, of valence type, and an amplification of a very low density hardly can lead to its saturation; ii) if indeed there was a peculiar, saturation-like effect observed in $x g$ at medium $x$, different from $x g$ as derived in $e p$ at HERA, then one would not know reliably whether that behaviour was resulting from the nuclear environment, a shadowing effect, or a genuine non-linear $g g$ interaction.
} 


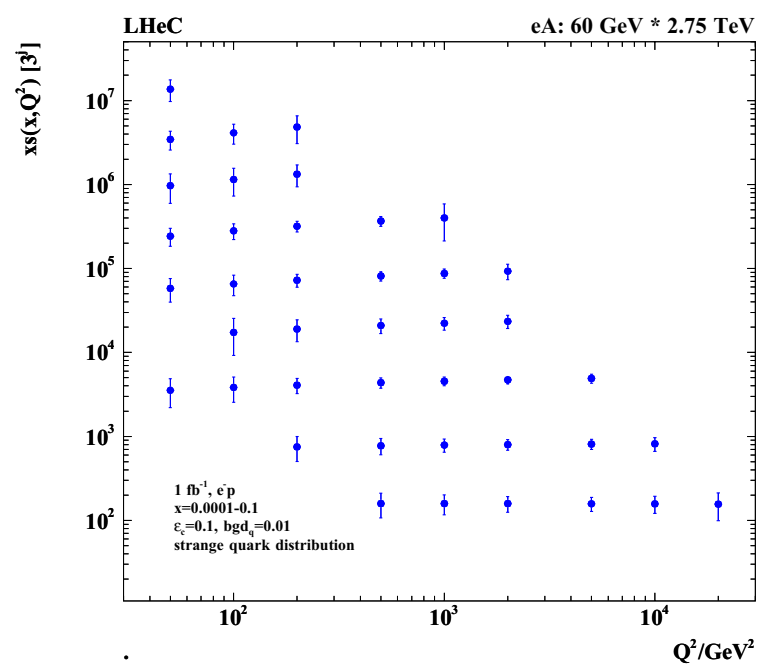

Figure 4. Simulation of the measurement of the (anti)-strange quark distribution $x \bar{s}\left(x, Q^{2}\right)$ in charged current $e A$ scattering through the t-channel reaction $W^{-} \bar{s} \rightarrow c$. The data are plotted with full systematic and statistical errors added in quadrature.

\section{Strange and Heavy Quark PDFs from the LHeC in eA Scattering}

The $\mathrm{LHeC}$ is the ideal environment to determine the strange, charm and beauty (also the top) PDFs. The principal technique is charm tagging (in $\mathrm{CC}$ for $x s$, in $\mathrm{NC}$ for $x c$ ) and beauty tagging (in NC for $x b$ ). The beam spot of the $\mathrm{LHeC}$ has the transverse extension of about $(7 \mu \mathrm{m})^{2}$. Modern Silicon detectors have a resolution of a few microns to be compared with typical decay lengths of charm and beauty particles of hundreds of $\mu \mathrm{m}$. The experimental challenges then are the beam pipe radius, coping at the $\mathrm{LHeC}$ with strong synchrotron radiation effects, and the forward tagging acceptance, similar to the HL-LHC challenges.

A study was made of the possible measurements of the anti-strange density (Figure 4) using impact parameter tagging in $e A C C$ scattering, and of the charm and beauty structure functions in NC (Figures 5, 6). Following experience on heavy flavour tagging at HERA and ATLAS, assumptions were made on the charm and beauty tagging efficiencies, to be $10 \%$ and $60 \%$, respectively. The light quark background in the charm analysis is assumed to be controllable to per cent level, while the charm background in the beauty tagging sample is assumed to be $10 \%$. The tagging efficiencies and background contaminations affect the statistical error. Moreover, an additional systematic error is assumed in the simulated NC (CC) measurements of $3(5) \%$. This results in very promising measurements of the heavier quark distributions: to about 10-20\% total uncertainty on the strange and 3-5\% on the charm and beauty measurements, for typically $x$ between $10^{-4}$ and 0.1 and $Q^{2}$ extending from below threshold $m_{Q}^{2}$ up to a few times $10^{4} \mathrm{GeV}^{2}$. The knowledge of the heavy quark densities is of prime relevance for understanding nuclear structure and the development of QCD as has often been emphasised. 


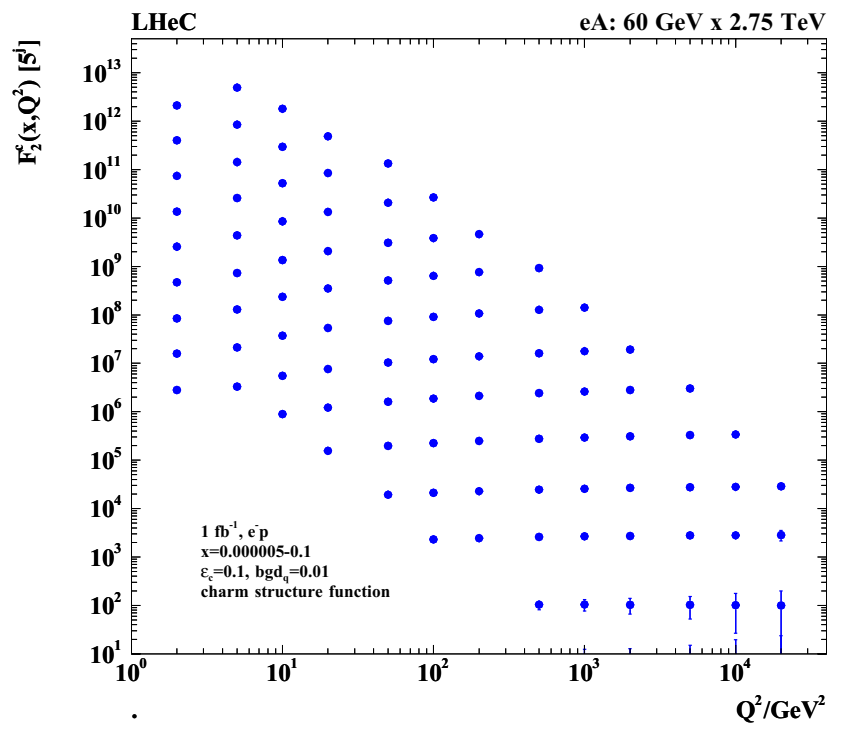

Figure 5. Simulation of the measurement of the charm quark distribution expressed as $F_{2}^{c}=e_{c}^{2} x(c+\bar{c})$ in neutral current $e A$ scattering. The data are plotted with full systematic and statistical errors added in quadrature.

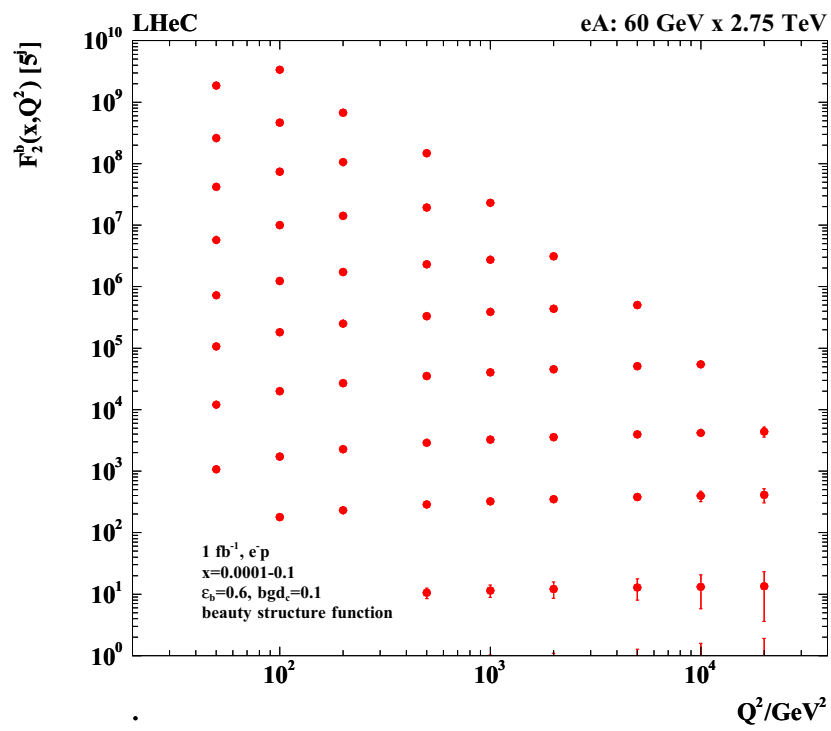

Figure 6. Simulation of the measurement of the bottom quark distribution expressed as $F_{2}^{b}=e_{b}^{2} x(b+\bar{b})$ in neutral current $e A$ scattering. The data are plotted with full systematic and statistical errors added in quadrature. 


\section{Summary}

The field of nuclear PDFs is in its infancy while the LHC probes heavy ion physics in $P b P b$ and $p P b$ highest energy collisions, surprisingly revealing similar collective phenomena. PDFs are determined in deep inelastic scattering. The $\mathrm{LHeC}$ will surpass the HERA information in various crucial areas: it will extend the kinematic range in $e p$ scattering by a factor of 20 , the luminosity by a factor of $10^{2-3}$ and provide data on electron-lead, and possibly electron deuteron, collisions which extend the current kinematic range in lepton-nucleus scattering by $3-4$ orders of magnitude. This programme will change the whole of nuclear substructure, parton dynamics and the QGP physics dramatically. It has been demonstrated here briefly how large the potential for a novel, complete and accurate determination of full PDF sets in $e D$ and $e A$ scattering is. The salient features of the $\mathrm{LHeC}$, in contrast to lower energy EIC configurations, are i) the very high $Q^{2}$ (and huge range) which makes weak neutral and charged currents a prime tool for separating different flavours in $e p$, en and $e A$ scattering leading to an unprecedented level of determining nuclear binding effects over 5 orders of magnitude in Bjorken $x$; and ii) the very low $x$ region covered in the perturbative domain which will discover the long awaited gluon saturation phenomenon, or further defer it to beyond sooner accessible ranges, to possibly the even larger energies considered now with the FCC-eh configuration. It is for these reasons and the here not covered huge variety of important nuclear physics phenomena accessible at energy frontier $e A$ scattering, partially presented at this workshop, that the $\mathrm{LHeC}$ has been put on the long range map for European nuclear physics [14] a few years ago.

Acknowledgement The present summary describes work jointly performed with Nestor Armesto, Peter Kostka, Hannu Paukkunen and Voica Radescu most gratefully acknowledged. Thanks are due to a large $\mathrm{LHeC}$ Collaboration of physicists and engineers engaged in the study of future deep inelastic scattering at the energy frontier. A special merci to the organisers of POETIC à Paris.

\section{References}

[1] J.C. Collins, D.E. Soper and G.F. Sterman, Adv. Ser. Direct. High Energy Phys. 5 (1989) 1.

[2] For a recent review see: H. Paukkunen, Nucl. Phys. A 926 (2014) 24.

[3] N. Armesto, H. Paukkunen, J. M. Penin, C. A. Salgado and P. Zurita, arXiv:1512.01528.

[4] T. Alexopoulos et al., eD Scattering with H1, A Letter of Intent, DESY 03-194;

H. Abramowicz et al., A New Experiment for HERA, MPP-2003-62;

M. Klein and T. Greenshaw, J. Phys. G Nucl. Part. Phys. 28:2505, 2002.

[5] J. Abelleira Fernandez et al., LHeC Study Group,

A Large Hadron Electron Collider at CERN, J. Phys. G Nucl. Part. Phys., 39(7):075001, 2012.

[6] LHeC Webpage: lhec.web.cern.ch.

[7] S.J. Brodsky, LHeC Physics Highlights, LHeC Workshop, Chavannes-de-Bogis, 2015, see [6].

S.J. Brodsky, I. Schmidt and J.J. Yang, Phys. Rev. D 70 (2004) 116003 [hep-ph/0409279].

[8] J. Blümlein and M. Klein, PHE-90-19, unpublished.

[9] M. Klein and V. Radescu, Partons from the LHeC, LHeC-Note-2013-002 PHY, Contribution to Snowmass 2013, unpublished.

[10] K. J. Eskola, H. Paukkunen and C. A. Salgado, JHEP 0904 (2009) 065.

[11] N. Armesto, presented at LHeC Workshop, Chavannes-de-Bogis, 2015, see [6].

[12] L. V. Gribov, E. M. Levin and M. G. Ryskin, Phys. Rept. 100 (1983) 1.

[13] K. Prytz, Phys. Lett. B 311 (1993) 286.

[14] NuPECC: Long Range Plan 2010: Perpectives of Nuclear Physics in Europe, www.nupecc.org 